\title{
COMPARISON FREE ENERGY BINDING SITES NEURAMINIDASE IN DIFFERENT DIELECTRICS \& TEMPERATURES , USING MOLECULAR DYNAMIC AND MONTE CARLO FOR NANO DRUG DESIGN
}

\author{
Maryam Mousavi ${ }^{1}$, Hooria Seyedhosseini Ghaheh ${ }^{1,2}$, Reza Rasoolzadeh ${ }^{1,2^{*}}$ \\ Mahmood Araghi ${ }^{3}$
}

1- Department of Biology, Science and Research Branch, Islamic Azad University, Tehran, Iran

2- Young Researchers and Elites club, Science and Research Branch, Islamic Azad University, Tehran, Iran

3- Department of pathology Amir - momenis Hospital ,Zanjan, Khodabandeh, Iran

\begin{abstract}
:
Neuraminidase (NA) is the essential surface glycoprotein of the influenza virus. High- affinity neuraminidase inhibitors have been designed that interact only with the conserved active site and binding site residues. The neuraminidase (NA) of influenza virus is the target of anti-flu drug.for treatment of this disease a thorough knowledge of neuraminidase protein is essential in order to produce potent drugs to suppress this enzyme..Drug design is by QSAR and docking methods, so we need a complete knowledge of receptor ligand, target site and binding site. This paper, using bioinformatics, Molecular Dynamics, Monte carlo and studied binding site NA enzyme in 310K temperature and different dielectrics $(1,78.39$ and 32.63) for the best drug designing. We measured the potential energy of amino acids binding to the drug. Molecular Mechanics, Molecular Dynamic and Nanobiological have done a great assistance in drug designing.
\end{abstract}

KEYWORD: Influenza A (H1N1), Drug design, Neuraminidase

\section{INTRODUCTION}

There have been worldwide Influenza in history of medication; the first epidemic one occurred in 1918 in Spain that about 50-100 million people died [1],the reason for this illness was H1N1. the second epidemic one accrued in 1957 in Asia that about 4 million people died, the reason for this illness was H2N2 the third epidemic one occurred in 1968 in Hong Kong and about 1 million people died and reason for that illness was H3N3.The importance of this illness is being epidemic and pandemic [2,3]. In Iran Aban 1388 , about 2662 cases with RT-PCR proved[4] ,Nowadays this illness is transmitted human by human and it can transfer very quickly by sneeze, cough and the secretion of nose and mouth, touching respiratory droplets [5]. This virus can be alive for 2 to 8 hours, so it can transfer by object, doors and public devices. The danger of transferring in the 
first four days is a fact [6]. we can recognize this illness by use of Nasofarnex and cell culture and also that Rt- PCR. that should interpret result in a good way[4].special patients and groups (high risk) such as : adults, younger children, pregnant women ,diabetics. Influenza A virus belongs to the Orthomyxoviridae family and is classified as three types A, B and C; the type A is clinically very important. The host range Influenza A is human, swine, hen, horses and birds[7,8,9]. The form of swine Influenza mostly spherical or ovoid and sometime as long spaghetti- like ,they are composed of lipid membrane, glycoprotein molecules HA and NA, and small amounts of the trans-membrane protein M2 [7,9]. The genome of influenza A viruses is eight segment of single strand RNA of negative polarity, that code about 11-12 protein[7,8].

\section{Neuraminidase inhibitor}

Zanamivir (Releza) and Oseltamivir (Tamiflu) are sensitive of Neuraminidase enzyme [10,11,12]. Amantadin and Rimantadin are resisting and target protein M2, consume of that forbidden by CDC $[4,13$

\section{EXPERIMENTAL}

In this study, data related to classification of Neuraminidase (EC3.2.1.18), the weight of subtype 200KD [14,15], and succession of amino acid in alpha and beta chain are gained in Protein Data Bank (2010) and NCBI site Then simulated the whole structure of enzyme by using of Argus Lab 4.0 software.

Since this protein is in PDB sites with inhibitor (Relenza=ZMR), simulated in link of active site that hosts cell hold in this site when there is not inhibitor Sialic acid, on the other hand is inhibitor binding site [16]. ( Sialic acid active site = Inhibitor site )

There are 4 amino acid involved with altering or drug that include: Asp151,Glu276,Arg152 and Arg371[9,14] .That introduced as binding site. This amino acid by hold of their situation stimulate with drug (ZMR). The phantom that gained of NA protein , proved that for amino acid have most interaction with drug in an active site .This amino acid has important role in drug design that is our studies in this search on binding site [17]. The structure data is related to Neuraminidase enzyme aspect of succession and quantity of amino acid provided quantity of water molecular for us. In structure of protein in Ca active site with drug by Argus Lab software .there are 7009 atoms in this protein, and our study is on 4 amino acid in this study binding site behave our and active site and enzyme was observed in aspect of potential energy level in different amino acid with drug studied in different solution such as Methanol, Water, Gas environmental like gas. Transferring of active enzyme to different parts aspect of dielectric constants is done by HyperChem 6.0 software. Then design of organic environmental is done by Chem3D software and considered fittest box aspect of X, Y, Z. The temperature is $310 \mathrm{~K}$ (37DC, body temperature), that studied the sites of involved enzyme with drug.

\section{RESULTS AND DISCUSSION:}

After Molecular Mechanic studies and gain potential energy by Monte Carlo method and studies of involved amino acid with drug in different dielectric constant, display as follow: 


\section{a) Binding site behavior in gas:}

A counting Molecular Mechanic of involved amino acid with drug in gas display like this, in primary stage step size 0 ps account in highest level $(90 \mathrm{kcal} / \mathrm{mol})$, that decrease of level energy $(51 \mathrm{kcal} / \mathrm{mol})$, by spending time, that in $10 \mathrm{ps}$ range ,the range of decreasing energy was observed clearly .The lowest level of energy is observed in 71-80pSec (Fig.1.a).

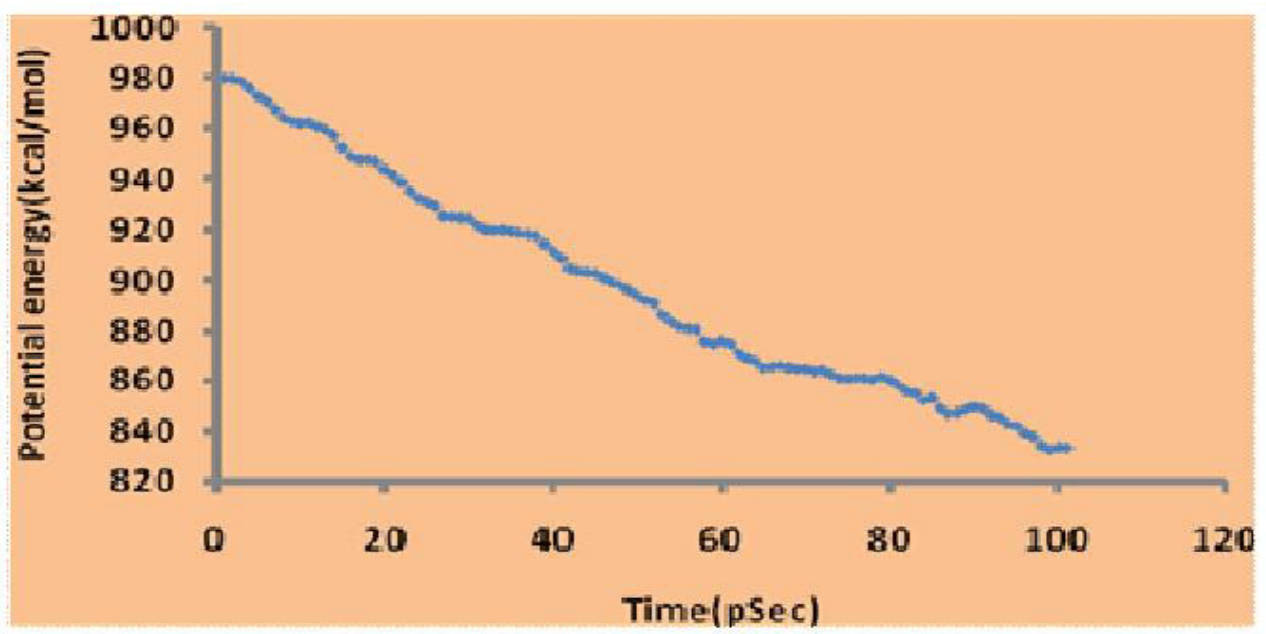

(a)

\section{b) Binding site behavior in water box:}

Accounting of Molecular Mechanic, involved amino acids with drug in water situation display the involved. Amino acids with drug enter to water part. The level of potential energy reached from $980 \mathrm{Kcal} / \mathrm{mol}$ to $833 \mathrm{Kcal} / \mathrm{mol}$ that is the figure of this accounts appear like this (Fig.1.b).

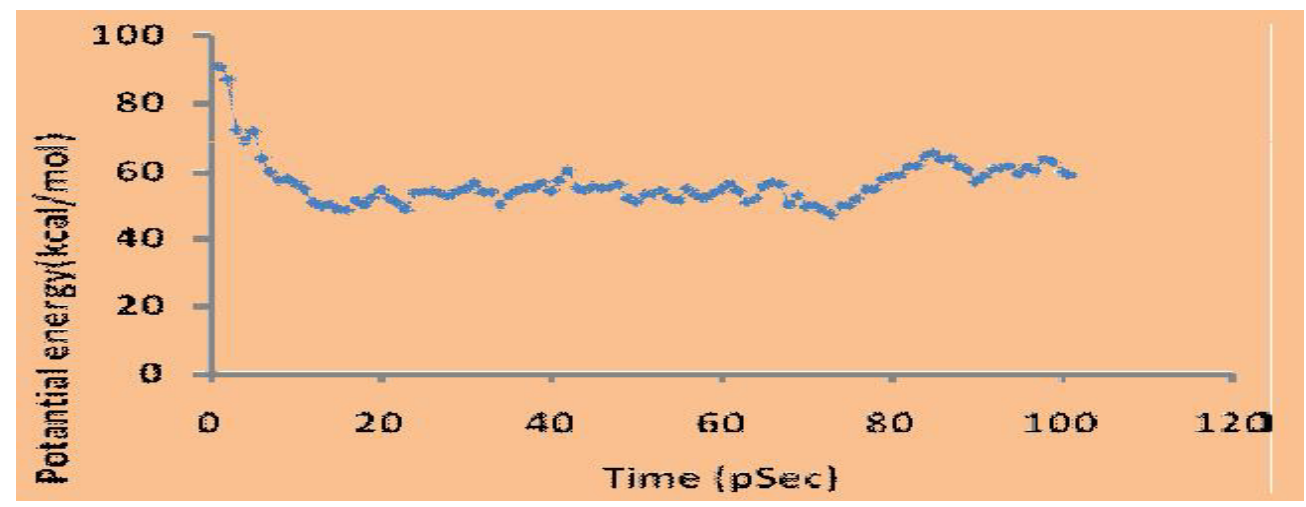

(b) 


\section{c) Binding site behavior in Methanol box:}

Accounts of potential energy of amino acid studied in Methanol part displayed that the level of energy during step size $0-100 \mathrm{ps}$ decreased from $11034 \mathrm{Kcal} / \mathrm{mol}$ to $1310 \mathrm{kcal} / \mathrm{mol}$. The figure of Monte Carlo confirms that passing time the level of energy has been decreased (Fig.1.c).

It shows that some drug synthesis in organic environment is expected some of this material enters to body with drugs. So one of the reasons that organic environment is biologic situation of body that protein's structure. Has hard and strong in this situation and is the best biological solution for correct synthesized and folding.(Tab1)

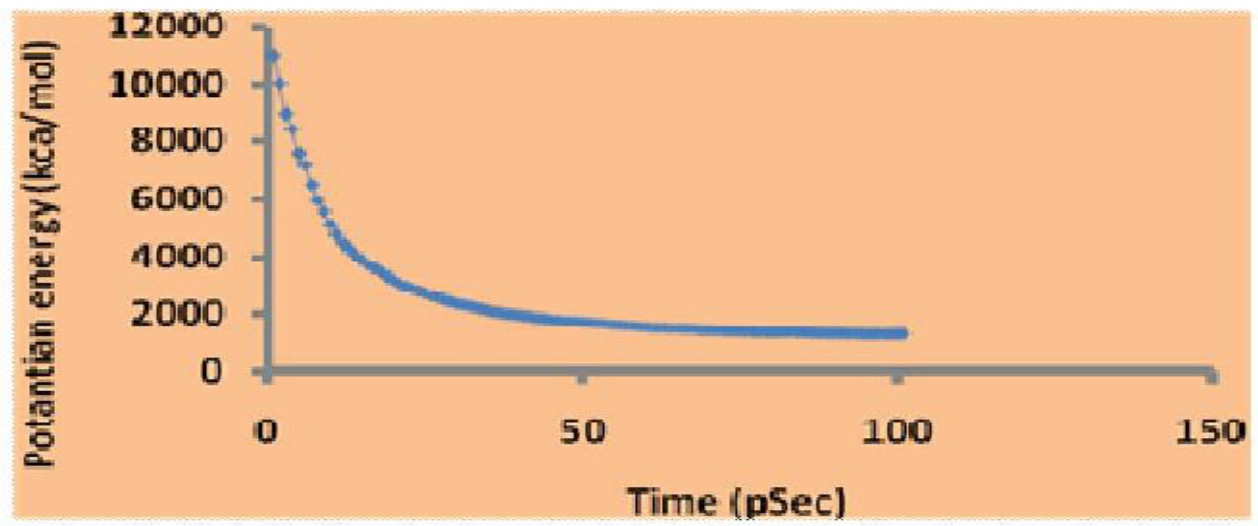

(c)

Fig.1.(a) potential of energy binding site NA in gas .(b) potential of energy binding site NA in Water box.

(c) Potential of energy binding site NA in Methanol.

\begin{tabular}{|c|c|c|c|}
\hline $\begin{array}{c}\text { Time } \\
(\mathrm{pSec})\end{array}$ & Water & Methanol & Gas \\
\hline 0 & 980.1198 & 11035.04 & 90.714 \\
20 & 941.8292 & 3058.884 & 53.85016 \\
40 & 908.946 & 1938.632 & 58.25608 \\
60 & 874.9766 & 1521.245 & 56.43553 \\
80 & 858.2182 & 1379.762 & 55.73374 \\
100 & 833.1655 & 1310.315 & 51.2155 \\
& & &
\end{tabular}

Table.1.comparison Potential energy (Kcal/mol) at $310 \mathrm{~K}$ in different dielectric

On the other hand most of drugs don't have any effect in water environment and the last one is that gas environment is base and important for druggist, infect all there studied by then dielectric constant are one of the most effective factors in determining molecular structure and biological function. Besides statistic studies like Quantum Mechanic and Molecular Mechanic help to develop in drug design, but for good understanding of drug design we should have full and complete data about receptor, target site and also binding site[18]. 


\section{RESULTS AND DISCUSSION:}

The study of binding site in gas shows that the system has the most and highest level of energy and lowest stability but by spending time the level of stability increase and the level of energy decrease, that is in put system that amino acid and aspect of them change by conformation, so have the highest stability, and in this condition binding site are in best condition, and gas part has negative effective on stability of binding site in Neuraminidase. Then in drug design has not effective[19]. Accounting of Molecular Mechanic (Monte Carlo) and comparing of amino acid stability is the main goal in designing drug and should this 4 amino acid has the first frame that require high stability or lowest level of energy .so this accounting proves this approach that water environment is very good for Neuraminidase in compare to organic and gas environment and in biological stability water part is good and fit for dielectric constants. Comparing of energy level in natural temperature and fever temperature. displays that when we face with Influenza enzyme it has significant change aspect of EPOT or Potential Energy and almost we can predict that stability or changes of enzyme behavior depends on dielectric constant not charge of temperature.Dielectric constant is one of most effective factors in determining molecular structure and biological function[20]. Besides statistic studies like Quantum Mechanic and Molecular Mechanic help to develop in drug design, but for good understanding of drug design we should have full and complete data about receptor, target site and also binding site. Their stability in temperatures $310 \mathrm{~K}$ has three solutions, water, and methanol and gas different dielectrics. Comparison of potential energy level binding site NA in Water part \& gas (Fig.2).

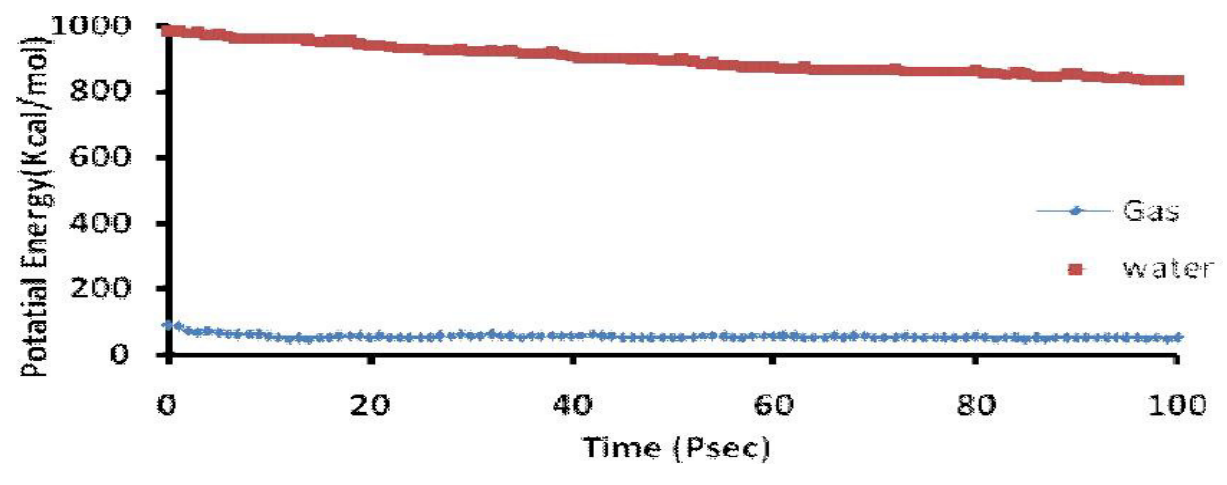

(a)

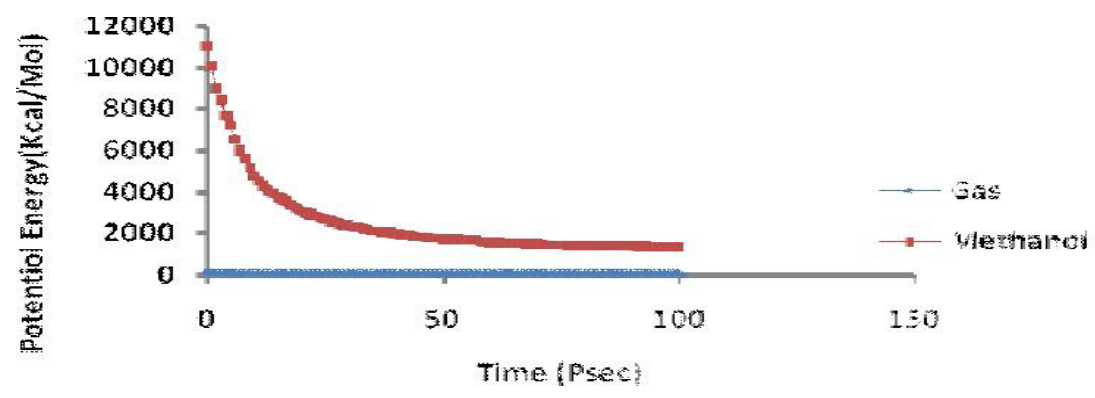

(b) 


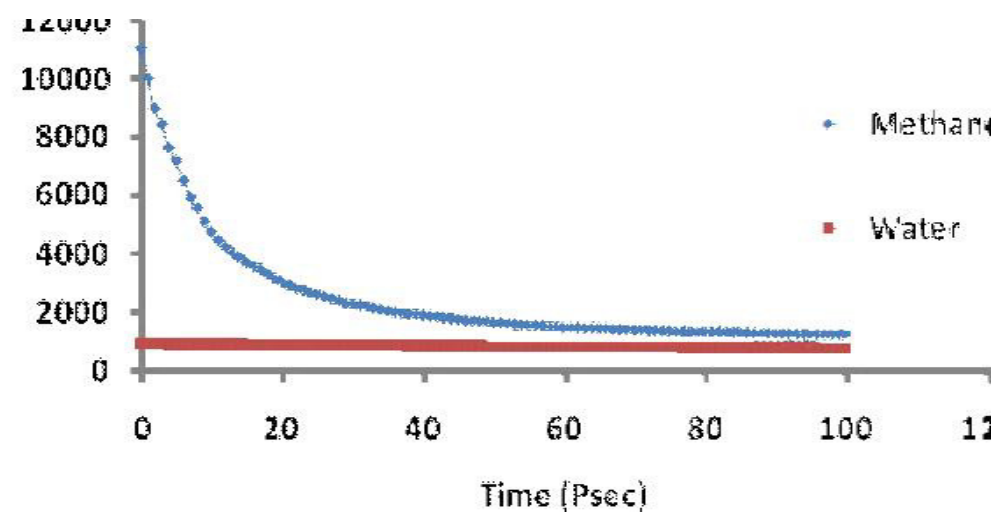

(c)

Fig.(2).a. Comparison of potential energy level binding site NA in Water part \& gas ,b. Comparison of potential energy level binding site NA in methanol part \& gas ,c. Comparison of potential energy level binding site NA in water part \& methanol part.

\section{REFERENCES:}

[1] Chen.C.Y, Huang.H.J,et al Drug design for Influenza A virus subtype H1N1.J.Taiwan institute of chemical engineers .(2010),41,8-15.

[2] Nguyen.T.N.A, Dao.T.T,et alInfluenza $\mathrm{A}(\mathrm{H} 1 \mathrm{~N} 1)$ neuraminidase inhibitors from vitis amurensis.J. Food chemistry.(2011), 124, 437-443.

[3] Wang.Y.T,Chan.C.H, Uansu.Z.Y,andLungchen .C.Homology modeling, docking, and molecular dynamics reveal HR1039 as a potent inhibitor of 2009 A(H1N1) influenza neuraminidase.J. biophyschem.(2010), 147,74-80

[4] Noorifard.M, Khoshdel.AR, Hosseini.SJ.A review in novel pandemic H1N1A.J.Army University of Medical Sciences of the I.R.Iran. (2009), 7, 228-240.

[5] Treanor .J.J. Influenza viruses including avian influenza and swine influenza, in mandell, Douglas and Bennett: principle and practice of infectious diseases.J. Philadelphia,Churchil livingstone. (2010), 7, 2265-88.

[6] Qi.Shi Du,et al. Computational 3D structures of drug-targeting proteins in the 2009-H1N1 influenza A virus. J. Chemical Physics Letters. (2010), 485, 191-195.

[7] Arias. C.F, Zamudio. M.E,et al. Molecular Anatomy of 2009 Influenza Virus A (H1N1). J. Archives of Medical Research. (2009), 40,643- 654.

[8] Gong. J,Wenfang.X.U.and Zhang.JStructure and Functions of Inflenza Virus Neuraminidase.J.Current Medicinal Chemistry.(2007), 14,113-122.

[9] Varghese. J.N. Development of Neuraminidase Inhibitors as Anti-Influenza Virus Drugs.J. Drug development research. (1999), 46,176-196.

[10] Adhiambo.M.O, Nguyen.H.T,et al.Host cell selection of influenza neuraminidase variants : Implications for drug resistance monitoring in A(H1N1) viruses. J.Antiviral Research .(2010),85,381388.

[11] Baranovich.T, Saito.R, et al.Emergence of H274Y Oseltamivir - resistant A(H1N1)influenza viruses in Japan during the 2008- 2009 season.J. Clinical virology . (2010),47,23-28.

[12] Roschek Jr.B,Fink.R.C, et al.Elderberry flavonoids bind to and prevent H1N1infection in vitro J.Phytochistry .(2009),70,1255-1261. 
[13] Bauer.K,Richter.M,Wutzler.PandM.Schmidtke.Differnet neuraminidase inhibitor susceptibilities of human H1N1,H1N2 and H3N2 infiuenza A viruses isolated in germany from 2001 to 2005/2006.J.Antiviral Research. (2009),82,34-41.

[14] Xiaojin. X.U, Xueyong. Z.H.U, Raymond. A.D, Stevens. J and Wilson. I.AStructural characterization of the 1918 Influenza virus H1N1 Neuraminidase.J.American Society for Microbiology . (2008),82, 10493-10501.

[15] Mercader.G.A, Pomilio.A.B. QSAR study of flavonoids and bioflavonoid as influenza H1N1 virus neuraminidase inhibitors. European Journal of Medicinal Chemistry . (2010),45,1724-1730.

[16] Wang. B., Dwyer.D.E.,et al.Detection of the rapid emergence of the H275Y mutation associated with oseltamivir resistance in severe pandemic influenza virus A /H1N1 09 infections.J. Antiviral Research. (2010),87,16-21.

[17] Lves.J.A.L, Carr.J.A, et al. The H274Y mutation in the influenza A/H1N1 neuraminidase active site following oseltamivir phosphate treatment leave virus severely compromised both in vitro and vivo. .J.Antiviral Research . (2002),55, 307-317.

[18] Lawrenz.M, Wereszczynski.J, etal.Impact of calcium on N1 influenza neuraminidase dynamics and binding free energy.J.Proteins . (2010), 78,2523-2532.

[19] Wang.S.Q, Shidu.Q, Huang.R.B,etal.Insights from investigating the interaction of oseltamivir (Tamiflu) with neuraminidase of the 2009 H1N1 swine flu virus.J. Biochemical and Biophysical Research Communications . (2009),386,432-436.

[20] Masukawa.K.M, Kollman.P.A, andKuntz.L.D. Investigation of Neuraminidase-Substrate Recognition Using Molecular Dynamics and Free Energy Calculations.J. Med. Chem. (2003),46, 5628-563 